\title{
Los saberes cívicos en la innovación de la gestión pública
}

Civic know-hows in the innovation of public management

\author{
Freddy Mariñez-Navarro / freddy.marinez@gmail.com \\ Tecnológico de Monterrey, Campus Monterrey, México
}

\begin{abstract}
This paper defines the concept of civic know-hows in the light of their components such as civic intelligence, civic engagement, social control and collaborative participation, as well as how their analysis is used to propose a kind of innovation in participatory governance. Through a theoretical exploration, we approach, on the one hand, the explanation of the elements of innovation in participatory governance, and on the other, the determination of the general meeting place in the complementary relation between the components of civic know-hows and the implementation of an improvement process or a new management, the introduction of a new service or improving the quality of an existing one, as well as the ways of providing them.
\end{abstract}

Key words: innovation, public management, civic know-hows, participation, collaboration.

Resumen: Este trabajo precisa el concepto de saberes cívicos a la luz de sus componentes tales como la inteligencia cívica, el compromiso cívico, el control social y la participación colaborativa; también el modo en que su análisis sirve para proponer un tipo de innovación participativa en la gestión pública. Mediante una exploración teórica, nos aproximamos, por una parte, a explicar los elementos de la innovación de la gestión pública participativa; y por otra, a determinar las líneas generales de encuentro en la relación complementaria entre los componentes de los saberes cívicos y la implementación de un proceso de mejora, o gestión nuevo, la introducción de un nuevo servicio o una mejora de la calidad en un servicio existente, así como las formas de proveer éstos.

Palabras clave: innovación, gestión pública, saberes cívicos, participación, colaboración. 


\section{Introducción}

Las nuevas condiciones socioeconómicas, políticas y culturales han impactado los enfoques sobre la innovación en la gestión pública. Para ello, los saberes cívicos - cuyos componentes son el compromiso cívico, la inteligencia cívica, el control social y la participación colaborativa- se han constituido en los elementos sustantivos de la modernización en la gestión de lo público. Partimos de la premisa de que se debe "innovar la Innovación en la Gestión Pública”, y ello es posible si la visualizamos como los arreglos de los actores gubernamentales, ciudadanos y otros actores no gubernamentales que permitan identificar problemáticas públicas, así como herramientas y dispositivos novedosos para lograr respuestas y soluciones nuevas en el espacio de lo público.

También, por otro lado, las modalidades democráticas de articulación de estos actores en un esquema de organización pública relacional reconfiguran la relación entre el Estado y la sociedad, generando el aprendizaje colectivo necesario para una gestión de calidad e inclusiva. En otras palabras, de lo que se trata es de construir redes de capacidades y de saberes donde se combinen recursos y expertise de varios actores para la coinnovación. Iremos infiriendo esta premisa expuesta en el transcurso del artículo cuando abordemos cada uno de los componentes de los saberes cívicos y la relación entre ellos.

Partimos de que estos saberes son todo lo que es y lo que sabemos de la convivencia democrática, de los derechos y obligaciones como ciudadanos, así como de las estructuras, funciones y medios del poder del Estado y del Gobierno. Implican la construcción propia del conocimiento ciudadano pertinente al grado de su cultura democrática. Es estar dispuesto a participar tanto presencial como a través de las tecnologías de la información y la comunicación (TIC) en el discurso racional, con la idea de ir generando un tipo de poder no delegado, desde los ciudadanos, donde su ejercicio establezca la cuestión de cómo las personas participan en la vida pública, esgrimiendo sus derechos frente a los gobernantes para la cocreación del valor público.

Visto así, proponemos que en el centro de la innovación de la gestión pública se ubican los saberes cívicos, pues los gobiernos tienden hoy a concebirse como plataformas democráticas participativas. ${ }^{1}$ Este trabajo prioriza

1 En la Carta Iberoamericana de Participación Ciudadana en la Gestión Pública (2009: 1) se afirma que "las sociedades contemporáneas de Iberoamérica demandan, cada vez con mayor fuerza, la ampliación y profundización de la democracia como sistema político y, en particular, la democratización de la gestión pública”. 
el enfoque analítico de los saberes cívicos a la luz de la visualización de sus componentes y el modo como su análisis sirve para proponer un tipo de innovación participativa en la gestión pública. Afirmamos que para innovar y hacer más legítima la innovación en la gestión pública se requiere involucrar democráticamente a los ciudadanos y actores no gubernamentales.

Los objetivos del artículo son: 1) analizar los componentes de los saberes cívicos como elementos legitimatorios de la innovación de la gestión pública participativa; y 2) determinar las líneas generales de la relación complementaria entre los componentes de estos saberes y la gestión pública. Haciendo una revisión teórica para ver cómo se ha discutido en la literatura el compromiso cívico, la inteligencia cívica, el control social y, sobre todo, la participación colaborativa, presentamos el marco de interacciones de estos conceptos, además de la manera en que se relacionan con la innovación en la gestión pública.

\section{Descubriendo los saberes cívicos para fortalecer la innovación en la gestión pública}

Para los efectos de este trabajo, concebimos a la innovación en la gestión pública como el reconocimiento de la creatividad y el resultado que imprimen los ciudadanos y actores no gubernamentales en su relación con los funcionarios públicos. Es decir, la participación de los ciudadanos es fundamental en esta visión para diferenciarlo del enfoque clásico de la innovación en la gestión sustentada sólo en los procesos y en el uso de herramientas tecnológicas como un fin (Mulgan y Albury, 2003; Windrum, 2008).

La innovación constituye, de esta manera, la implementación de un proceso de mejora, o gestión nuevo, relativo a tres ámbitos: 1) el producto, bien o servicio (introducción de un nuevo servicio o una mejora de la calidad en un servicio existente); 2) el proceso operativo (alteraciones o cambios en las formas de proveer servicios públicos); y 3) la organización o proceso de gestión (cambios en las estructuras organizativas, en las prácticas de gestión y en las rutinas) (OCDE, 2012).

En este sentido, la eficacia y la eficiencia de las organizaciones públicas se han venido buscando a través de la mejora de la calidad y de la innovación gubernamental sustentada en los siguientes pilares de la calidad total: la satisfacción de los clientes, la mejora continua de los procesos y el desarrollo del personal. La aplicación de este concepto dio como resultado el reacomodo del arreglo tradicional de las organizaciones, induciendo cambios como el 
privilegio de los resultados financieros de las empresas y de los gobiernos, desplazándose hacia la calidad de los productos, enfatizándose en el sector público en la eficiencia económica de las operaciones y el equilibrio riguroso de las finanzas públicas; también hizo énfasis en la productividad, moviendo la atención de los productos hacia los clientes, generando que el producto sea un componente integrado del servicio, elevándose la productividad a la dimensión de competitividad por la calidad de los productos.

Por otro lado, vemos que la administración hacia adentro, que caracterizó a la administración weberiana, se ha extrovertido hacia los usuarios de los bienes y servicios de la organización, poniendo el punto final de una administración pública sin público y un gobierno sin ciudadanos (Aguilar, 2006).

A diferencia del concepto de innovación gerencial anteriormente explicado, partimos de un concepto de innovación en la gestión pública donde el centro del análisis sean los componentes de los saberes cívicos, expresado en que el trabajo contenido en aquella es realizado tomando como base el compromiso de los ciudadanos con el gobierno, facilitando la inteligencia cívica, el mejoramiento del control de la gestión vía la contraloría social, así como una concepción compartida de lo público, entendida como competencias de colaboración para el logro legitimado de la eficiencia y la eficacia de los gobiernos.

En este sentido, presentamos la innovación en la gestión pública atendiendo a dos direcciones: 1) a los arreglos de los ciudadanos, actores gubernamentales y no gubernamentales que permiten identificar problemáticas públicas, además de herramientas y dispositivos innovadores para el logro de respuestas y soluciones nuevas en el espacio de lo público; 2) como consecuencia de la anterior, a las modalidades democráticas de articulación de estos actores en la organización pública, produciendo una nueva configuración entre el Estado y la sociedad que asegura el aprendizaje colectivo y los saberes cívicos necesarios para una gestión de calidad e inclusiva.

Sólo así vemos a las organizaciones públicas más relacionales, a la gestión pública más participativa y a las políticas públicas, en todo su ciclo, más deliberativas en la construcción de la agenda, más incluyentes en aportar soluciones (diseño), y más colaborativas por los múltiples actores en acción en la implementación (Peters, 2014). Todo ello para lograr la satisfacción ciudadana que es definida, según Im y Lee (2011: 420), como "la combinación de las evaluaciones generales y subjetivas de las experiencias que tiene la gente con los servicios públicos, que incluyen no sólo la sensación u opiniones sobre los 'servicios objetivos', sino también evaluaciones de juicios". 
Las características diferenciadoras de la innovación con base en los saberes cívicos y la innovación gerencialista podemos sistematizarla de la siguiente manera. Mientras que en la primera el tipo de satisfacción se relaciona con la de los ciudadanos, en la innovación gerencial la satisfacción es del cliente, mediante la mejora continua de los procesos, productos y servicios. Otra característica es el aprendizaje.

La innovación con participación en la gestión genera un tipo de aprendizaje colectivo entre funcionarios y ciudadanos, dado al tipo de organización pública relacional, flexible y sobre todo inclusiva. En la innovación gerencialista, el aprendizaje es sólo entre los funcionarios públicos, lográndose un aspecto relacional, pero no inclusivo. En cuanto a la participación en la gestión, en la innovación con saberes cívicos, los funcionarios públicos crean oportunidades para comprometer a los ciudadanos, usando estas oportunidades para educar, organizar y activar la defensa de la participación ciudadana, creando un criterio de legitimidad basado en la coproducción de la innovación; mientras que la innovación gerencialista da sólo un marco orientado al mercado en la entrega al "cliente" del servicio público o producto, y su única legitimidad son los resultados (véase Cuadro 1$)^{2}$

En este contexto, innovar en la gestión pública debe ir de la mano con el fortalecimiento tanto de las instituciones democráticas (Rizvi, 2008) como de las relaciones societales, y de éstas con el gobierno. "Hay innovación cuando ciertos tipos de problemas no tienen solución en las instituciones existentes. Los actores deben conseguir soluciones innovadoras de sus problemas dado a la insuficiencia institucional" (Harrison y Klain, 2007: 8). Así, resolver esta falencia no se hace más que apelando a los saberes cívicos con el fin de completar y expandir las capacidades del sector público. En otras palabras, construir redes de capacidades y de saberes en las que se combinen recursos y expertise de varios actores para la coproducción (Fung, 2008).

Con el objetivo de reunir las demandas de la sociedad, una gestión pública novedosa requiere de funcionarios públicos emprendedores para organizaciones que se preparen para la diversidad, la adaptación y la reinvención. Vemos, en este sentido, que el emprendimiento como clave de la innovación basado en saberes cívicos ayuda a realizar y tomar ventajas de oportunidades para que las organizaciones públicas mejoren su implementación. Mothae y Sindane (2013: 117) lo definen como "el desarrollo de nuevos impulsos en el contexto de todo lo amplio de las organizaciones públicas. Funcionarios

2 Los cuadros se encuentran en el Anexo, al final del presente texto (Nota del Editor). 
públicos a través del emprendimiento, se convierten en emprendedores en sectores de sus organizaciones".

En otras palabras, como refieren Goldsmith et al. (2010), se requiere de un emprendimiento representado en el espíritu de cambio y el espíritu de comunidad (virtud cívica).

Los saberes cívicos en la innovación de la gestión pública se convierten en los aspectos sustantivos de la innovación misma. Para que ésta se lleve a cabo, se requiere de sus cuatro componentes relacionados entre sí, doblemente conectados con la democracia. Por una parte, a los procesos de innovación democrática de la gestión pública como parte de la democratización del sistema político y, por la otra, al derecho de participación que tienen los ciudadanos en la gestión pública. Analizaremos a continuación con más precisión cada uno de los componentes de los saberes cívicos.

\section{Compromiso cívico}

El primero de los saberes cívicos es el compromiso cívico. Se le entiende como la forma en que los ciudadanos, a través de una obligación o promesa, logran impulsar el asociacionismo con la idea central de llevar a cabo el debate, la deliberación y la solución de problemas públicos. Se trata de un punto más allá de la participación ciudadana que asegura que toda la gente haga uso de sus derechos de libertad, pues éstos y la ciudadanía, según Barber (2004), son correlativas, cada una sostiene y da vida a la otra.

No importa la seguridad, los derechos individuales y la autonomía que disfruten: los hombres y mujeres que no se hacen directamente responsables de las políticas que determinan sus vidas a través de la deliberación, la decisión y la acción comunes no son en absoluto libres. Y en este orden, Subirats (2015: 4) nos precisa que:

los debates sobre la gestión de recursos, la gestión de servicios, el gobierno y la gestión de las instituciones, toman otro sentido si se parte de las siguientes premisas: construir una nueva política local desde los ciudadanos, desde los barrios, desde los expertos y saberes ciudadanos, desde los políticos-ciudadanos, desde los que saben por qué y han padecido y luchado contra los efectos de las decisiones que han ido tomando los que aseguraban que sabían lo que hacían y que lo hacían por nuestro bien.

Nos referimos entonces a las diversas formas de diálogo ciudadano que son altamente inclusivas y a la deliberación para la hechura de las políticas y decisiones públicas. Esto es, "la clave de la virtud deliberativa como la reciprocidad, es decir, argumentar en términos que otros pueden aceptar" 
(Gutmann y Thomson, 1996, citado por Dryzek, 2009). La inclusión es un aspecto importante cuando hablamos del compromiso cívico, pues tiene como objetivo proporcionar a las personas un sentido de dirección para que las actividades asociativas y comunitarias tengan voz en su relación con el gobierno y así ayudarle a mejorar sus decisiones. El éxito del compromiso cívico con el gobierno requiere de innovación (Fung, 2008).

Así, el compromiso cívico ciudadano le da soporte efectivo al funcionamiento de la democracia, a la calidad del gobierno y a la inclusión en la administración pública, generando contextos para la legitimidad, el éxito de la implementación de la política pública y el logro de resultados sociales: valor social y valor público.

Compromiso ciudadano, para nuestra definición, significa algo más que la participación ciudadana. La diferencia radica en el modelo de producción de gobernanza. Participación ciudadana se da en un modelo de producción tradicional de gobierno donde los ciudadanos son actores o partes interesadas que actúan en un papel de consulta para y con las instituciones establecidas. Compromiso ciudadano sale de un modelo de coproducción de la gobernanza donde los ciudadanos son una parte esencial del proceso de producción. Ciudadanos comprometidos están, en teoría, comprometidos con algún sentido más amplio del "bien común", más allá de sus intereses individuales (Simrell y Martinelli, 2005: 2).

La visualización de Simrell y Martinelli (2005) es pertinente ya que el compromiso de los actores gubernamentales y no gubernamentales, y a nivel organizativo en sus asociaciones en la vida pública, es una amplia tendencia en la gestión pública. Esto pone en el centro del debate el rol de los funcionarios públicos en este compromiso. Hoy vemos en dicha tendencia el paso de un funcionario público experto-neutral a un facilitador de la participación y el compromiso. "En este escenario, los administradores públicos crean oportunidades para comprometerse con los ciudadanos y utilizar esas oportunidades para educar, organizar y activar a los ciudadanos a defender y participar en una esfera pública más amplia” (Brainard y McNutt, 2010: 841).

De este modo, el compromiso cívico también se expresa como un proceso de comunicación directa, tanto presencial como a través de las tecnologías de información disponibles, y mediante las cuales la gente discute de manera abierta sus razones, intereses, habilidades y valores, con la intención de llegar a un acuerdo para la toma de decisión. "[El] compromiso cívico ciudadano en las redes sociales permite a los individuos expresar sus intereses y demandas al gobierno. También permite que las voces de los individuos sean escuchadas, conduciendo a una mayor inclusión. Las redes de compromiso cívico también hacen a los ciudadanos más competentes" (Michels, 2011: 278). 
También Bailur y Gigler (2014) parten de que el compromiso cívico implica el empoderamiento ciudadano, por lo cual se necesita de los recursos de la participación, la transparencia y la rendición de cuentas. Por un lado, la participación les hace elevar sus requerimientos y voces (con la idea de que sean escuchados). En este sentido, las herramientas Web $2.0^{3}$ proveen una oportunidad para empoderar a los ciudadanos porque bajan los impedimentos para participar. Con dichas herramientas éstos pueden acceder directamente a la información y comunicación en lugar de ser dependientes de actores intermediarios.

Por otro lado, la transparencia es un recurso importante para el empoderamiento porque se trata de cualquier intento (por el gobierno o ciudadanos) de colocar información o proceso que antes eran opacos en el dominio público para el uso de los grupos ciudadanos, proveedores o hacedores de política. Y el tercer recurso del empoderamiento es la rendición de cuentas (accountability), entendida como la relación entre el poder titular (proveedor titular) y el poder delegado (los demandantes). Se requiere normas establecidas para adquirir información sobre acciones y su pertinencia, hechura de las decisiones, y la identificación y sanción de resultados insatisfechos.

Heeks (2000, citado por Bailur y Gigler, 2014) asume que las TIC (Web 2.0) permiten que el empoderamiento esté basado en las condiciones siguientes: a) la disponibilidad y transparencia de los datos; $b$ ) esta información debe ser accesible por los hacedores de políticas públicas, quienes son capaces de evaluar y transformar los datos en información; c) la información es usada para iniciar el diálogo y el activismo entre ciudadanos-gobierno y ciudadanos-ciudadanos; y d) el gobierno toma acciones con base en estos procesos.

Con base en lo planteado anteriormente, podemos precisar más sobre el compromiso cívico y el rol fundamental de la Web 2.0 en la innovación en la gestión pública:

1. La Web 2.0 y las redes sociales son cambios en las formas de interactuar la sociedad y el gobierno.

3 El término Web 2.0 fue acuñado en 2005 por Tim O’Reilly, quien define los siete principios constitutivos de las aplicaciones Web 2.0: la Web como plataforma; el aprovechamiento de la inteligencia colectiva; la gestión de la base de datos como competencia básica; el fin del ciclo de las actualizaciones de versiones del software; los modelos de programación ligera junto a la búsqueda de la simplicidad; el software no limitado a un solo dispositivo; y las experiencias enriquecedoras de los usuarios. 
2. La información es difundida a través de múltiples canales, permitiendo que los ciudadanos la reciban en el momento y lugar que ellos quieran, y puedan responder.

3. Los gobiernos necesitan considerar la Web 2.0 como un camino para llegar a ser relevante a sus ciudadanos. En la innovación en la gestión pública sustentada en saberes públicos, los gobiernos tienen nuevas herramientas para escuchar más fácilmente esa voz.

4. Se logra una gran conectividad que permite a los ciudadanos encontrar que cada uno cree comunidades de intereses alrededor de temas que son importantes para ellos.

5. El gobierno puede actuar como agente creativo mediante la apertura de datos y acceso a la información gubernamental.

\section{Inteligencia cívica}

El otro componente es la inteligencia civica, vista como inteligencia colectiva directa dirigida a enfrentar los desafíos compartidos. Es decir, dirigir las capacidades percibidas, actuales y potenciales de los individuos, grupos y organizaciones cívicas para responder inteligente, efectiva y equitativamente a los desafíos actuales, con el fin de lograr el desarrollo de la convivencia democrática. La inteligencia cívica no es más que tener información y comprenderla para compartirla a quienes necesitan respuestas.

En otras palabras, implica conocer, responder y proveer, por lo que se podría entender como la capacidad de las organizaciones ciudadanas y comunitarias de adquirir y aplicar conocimientos; o bien, como la capacidad para adaptar efectivamente, o simplemente para la resolución de problemas, aprendizaje, razonamiento, predicción, reflexión e imaginación de los ciudadanos (LaDuke, 2008). No es más que la lógica de la cocreación de soluciones, definición de problemas sociales y de colaboración. Por eso el crowdsourcing juega un papel importante en la inteligencia cívica, ya que los ciudadanos aprovechan internet para adquirir recursos, conocimiento, experiencia o tiempo (colaboración abierta).

Zhao y Zhu (2012, citado por Seltzer y Mahmoudi, 2012) definen el crowdsourcing como un sistema de inteligencia colectiva. Y Surowieck (2005, citado por Seltzer y Mahmoudi, 2012: XIII) habla de la sabiduría del público, "en las circunstancias adecuadas, los grupos son muy inteligentes, y con frecuencia son más inteligentes que las personas más inteligentes del grupo”. En muchos países no desarrollados, el crowdsourcing es aplicable en el marco de 
consultas populares, monitoreo de elecciones, procesos de redacción de Constitución, o en cualquier lugar que se asegura de que las voces de la diversidad étnica y política y grupos minoritarios serán escuchados (Bott et al., 2014).

Schuler (2010) nos presenta una definición de inteligencia cívica cuyo punto de partida son los diversos tipos de proyectos ciudadanos y comunitarios para motivar y coordinar diversos esfuerzos que se refieren a cómo esta inteligencia se encuentra resolviendo sus problemas comunes, además de que grupos de personas persiguen fines cívicos a través de medios cívicos. Para este autor, dicha definición incluye: a) la evidencia cuando los grupos trabajan hacia un fin explícito; $b$ ) el aprendizaje para el involucramiento ciudadano; c) la movilización de los grupos ciudadanos alrededor de desafíos compartidos; d) el modo en que los grupos de ciudadanos piensan y actúan; e) la formulación de compromisos en lo meta-cognitivo, es decir, se piensa sobre su pensamiento; $f$ ) la organización de los ciudadanos y sus proyectos con una orientación crítica.

Además, Shuler (2008: 81) precisa el concepto de inteligencia cívica como la "capacidad de los grupos y organizaciones, e idealmente la sociedad en su conjunto, para concebir y poner en práctica estrategias eficaces, equitativas y sostenibles para compartir problemas".

Sin duda, la inteligencia cívica, como ejemplo de innovación social y saber cívico, es una respuesta a una necesidad percibida donde la recepción que obtiene o no, por parte de los ciudadanos, va en proporción a la necesidad de percepción por éstos.

\section{Control social}

El tercer componente de los saberes cívicos es el control social, que no es más que la participación ciudadana orientada al monitoreo, la vigilancia y la evaluación por parte de personas u organizaciones sobre programas y acciones gubernamentales, incidiendo directamente en la esfera pública, ya sea formalmente por medio de su inclusión en comités de obras, órganos colegiados, etc., o indirectamente por medio de la activación de los sistemas de quejas y denuncias (Hevia de la Jara, 2006). El control social es un tipo de control que en vez de originarse desde las instituciones administrativas y políticas, emana desde la sociedad, el control directo desde los ciudadanos a los políticos y funcionarios públicos.

Hoy tenemos un nuevo marco de análisis para el control social a partir de las herramientas Web 2.0. Mediante el software social se logra una serie 
de aplicaciones que pretenden ampliar las posibilidades de comunicación y de conexión entre personas que ya permiten el e-mail o los sistemas de mensajería instantánea. Son herramientas colaborativas que une a una serie de personas con el fin de facilitar el flujo de información (agendas, mensajes, denuncias) y la realización de trabajos conjuntos entre ellas (colaboración en el monitoreo o vigilancia de las políticas públicas).

En consecuencia, partimos de cuatro ejes que hacen al control social más efectivo:

Primer eje: transparencia colaborativa. Debe existir un proyecto de datos abiertos, aplicado como una filosofía y forma de administración, donde todos los datos públicos estén disponibles y actualizados en un formato libre, gratuito y sin ninguna restricción. No sólo se ofrece transparencia a la gestión, sino acceso a datos comunes y necesarios que pueden ser utilizados por toda la población para diversos fines, como información de índole económica, estadística, meteorológica, geográfica o turística, así como la social, la salud y la educación, y series de datos que no pueden ser obtenidos fácilmente. Además, se alienta la colaboración, el trabajo conjunto de las organizaciones o particulares con los datos oficiales y la participación de las personas como auditores implícitos de la gestión.

El segundo eje se relaciona con la organización y las capacidades de los ciudadanos. Precisar la organización y la capacidad ciudadana en el marco de las herramientas 2.0 para el control social, requiere que los ciudadanos logren la legitimidad técnica para poder hacer uso de las plataformas software social y sus herramientas de colaboración que permiten comunicar, coordinar, compartir recursos, aplicaciones y contenidos. Es importante destacar que en este contexto los ciudadanos deben empoderarse para poder realizar las actividades de colaboración para el control social.

Pero, ¿cómo pueden permitir las Web 2.0 el empoderamiento de los ciudadanos? Por una parte, con los flujos de información de arriba hacia abajo: desde el gobierno a los ciudadanos. Por la otra, la creación de la posibilidad de flujos de abajo hacia arriba: de los ciudadanos con el gobierno, que son esenciales para la toma de decisiones. Y por último, los flujos de comunicación horizontales, tales como las redes interorganizacionales y las redes sociales. Estamos hablando de un control social en red y no jerárquica.

El tercer eje es el acceso a la información. Partimos de la base de que el acceso a la información es un derecho ciudadano reconocido en muchos países a nivel constitucional. También vale destacar que sin información pública disponible los ciudadanos no tendrían la capacidad para vigilar y monitorear 
la gestión de los gobiernos y de sus políticas. Esta es la razón por la cual debe ser garantizada y completa, así como no distorsionada ni atrasada y sin altos costos; ello permitirá minimizar problemas de asimetría de la información.

Y el cuarto y último eje lo relacionamos con lo público. El control social no sólo se aplica al gobierno, sino a todos los actores que intervienen en la producción del valor público. Saxton y Guo (2011) han venido afirmando con base en sus investigaciones que con la difusión de las tecnologías de internet, aparecen dos dimensiones fundamentales de la rendición de cuentas de las organizaciones civiles: la divulgación y el diálogo. Quiere decir que si la producción de lo público es colaborativa (cocreación), los diversos actores (gubernamentales y no gubernamentales, y ciudadanos) son objeto de ser controlados por sus acciones.

De una manera más precisa, el control social es el medio para ejercer el monitoreo, la fiscalización y la vigilancia de la ejecución del plan de desarrollo de la política pública y el servicio público, permitiendo, por una parte, prevenir a tiempo irregularidades, desviaciones de los recursos destinados a obras, servicios, programas y proyectos a los actores de la cocreación pública, y sugiriendo cómo corregirlas. Por otra parte, contribuye al cumplimiento de las leyes y procedimientos en la ejecución de las obras, servicios, programas y proyectos financiados con fondos públicos, y, por último, incentiva la confianza ciudadana en la incorporación social a la vigilancia sobre los recursos destinados a satisfacer las necesidades de la colectividad. "Además, el control social surge como una forma sustancialmente diferente de los modelos tradicionales de control: político, fiscal, judicial, interno, disciplinario; pero igualmente es un escenario de interacción entre el Estado y la sociedad civil" (Garcés, 2006: 480).

El control social no es posible sin el compromiso cívico, la inteligencia cívica y la participación colaborativa, ya que sus objetivos están orientados a garantizar a los ciudadanos el acceso a la información, impulsar la participación y la corresponsabilidad; mejorar la eficiencia, la eficacia y la confiabilidad de las acciones gubernamentales, e incorporar a la ciudadanía a ser vigilante de la corrupción.

\section{Participación colaborativa}

El cuarto y último componente de los saberes cívicos lo identificamos con la participación colaborativa, entendida como el cotrabajo basado en el valor de la reciprocidad, pudiendo incluir al público (Agranoff y McGuire, 2003). 
En otras palabras, las capacidades de los ciudadanos, las empresas y el mismo gobierno para aportar en la solución o en la gestión de los asuntos públicos, tomando necesariamente en cuenta el uso de las TIC.

Es evidente que para que la participación colaborativa se cristalice y se convierta en un factor de innovación en la gestión pública, es menester la existencia de una política gubernamental que posibilite un mayor acercamiento a través del diálogo entre gobierno y ciudadanos, dando así cabida a la atención y gestión de las demandas, la negociación e interlocución entre ellos, y la colaboración.

Harrison et al. (2011) afirman que la deliberación y la participación deben dar la oportunidad para el intercambio de visiones con el objeto de determinar el curso de acción para asegurar que diversos puntos de vista de ciudadanos, grupos civiles y actores no gubernamentales sean incorporados a la acción del gobierno. Es decir, la participación colaborativa se da en el momento en que los individuos son parte integrante, con su expertise, junto con el gobierno, del proceso de cocreación de las decisiones para generar soluciones que luego serán implementadas. Welch (2010, citado por Meijer $e t$ al., 2012), sugiere que "la participación puede ser más importante en las etapas iniciales del ciclo de las políticas públicas, mientras que la transparencia se convierte más relevante en su implementación. Los ciudadanos quieren participar para influir y monitorear las políticas mediante el acceso abierto a la información" (Meijer et al., 2012: 15).

La participación colaborativa implica un cambio en el juego de las legitimidades políticas, técnicas, sociales y éticas de los ciudadanos y otros actores no gubernamentales, por lo que nos lleva a ver la lógica gubernamental operando con esquemas de cocreación, pues ésta:

envuelve dinámicas innovadoras para encontrar soluciones de una manera poco convencional, ya sea a través del desarrollo de una nueva solución o un nuevo enfoque que incorpora al mismo tiempo una variedad de actores. Lo distintivo de la innovación en procesos participativos de la cocreación, por lo tanto, no se relaciona sólo con una nueva técnica sino con una forma de generar nuevo conocimiento de manera colectiva (Zurbriggen y González, 2014: 339).

La participación colaborativa impacta a la innovación en la gestión pública en el momento en que abre una posibilidad de diálogo, comunicación, legitimidad y confianza, convocando a los diferentes actores de la sociedad a trabajar juntos, reconociendo que los ciudadanos poseen información propuesta por diversos actores que puede ser usada para resolver los problemas públicos. "Un nuevo paradigma de la colaboración en la administración pú- 
blica requiere que ciertos comportamientos en línea sean aprendidos, comprendidos y adoptados" (Edelmann et al., 2012: 22).

\section{La lógica de los saberes cívicos en el proceso de colaboración}

Michels (2011: 280) distingue cuatro tipos de innovaciones democráticas que pueden mejorar la gestión pública colaborativa y participativa:

a) El referéndum, que da a los ciudadanos un voto directo en las decisiones políticas;

b) la hechura de la politica pública participativa, que se caracteriza porque hay una clara relación con la toma de decisiones en el sentido de que se les pide a los ciudadanos y a las partes interesadas asesorar al Gobierno; c) las encuestas deliberativas, que son las opiniones individuales en el que se le pregunta al ciudadano acerca de un tema en particular, y d) los foros deliberativos, entendidos como foros dialógicos diseñados con el objetivo de ser deliberativo, lo que significa que la atención se centra en el seguimiento de los procedimientos deliberativos ideales, la formación de la opinión y el intercambio de argumentos tan importantes en la toma de decisiones.

Para el interés de la participación colaborativa en la innovación de la gestión pública, entendemos a la participación como la acción por medio de la cual se dirimen problemas específicos para encontrar soluciones comunes o para hacer confluir voluntades dispersas en una sola acción compartida (colaboración en el proceso y hechura de la política pública y decisión pública). Entonces, al hablar de las decisiones públicas, estamos ubicando la participación en el sentido de tomar parte en la gestión de lo colectivo, de la cosa pública. De esta manera, la participación es sinónimo de intervención, de interés, de preocupación que supone un valor agregado de voluntad, un sentimiento de pertenencia a un colectivo, a un grupo, a un municipio, a una entidad federal, a un país. También es importante afirmar que los ciudadanos y las organizaciones que participan en la esfera de la sociedad civil necesitan conectarse con otras parecidas en una red de contactos que permita construir contenidos, intercambiar información y experiencias, y llevar a cabo proyectos comunes.

Esta afirmación ubica a los ciudadanos y a la sociedad civil como sede donde se pueden encontrar las nuevas fuentes de legitimidad y los nuevos espacios de consenso basados en la tolerancia y el debate, la colaboración y el diálogo. Este último, entendido como un medio por el cual se canaliza de manera racional la pluralidad ideológica, se convierte en clave para la participación, ya que a través de ella varios actores políticos y gubernamentales y ciudadanos pueden expresar sus ideas, creencias, investigaciones, conclusio- 
nes, experiencias, etc., con la finalidad de que puedan llegar entre todos a un acuerdo y, como punto final, tomar una decisión, que afectará de manera positiva/negativa o directa/indirecta a la política gubernamental (Barber, 2004).

Entonces, los gobiernos con visión pública moderna pueden enfrentar el reto de la participación: "dar forma a las interacciones entre los múltiples actores que participan en la identificación, el diseño y la puesta en práctica de una política. Es decir, el reto actual de la modernización administrativa no es mejorar la competencia y la eficiencia de las organizaciones, sino modificar su mentalidad: sustituir la lógica del cada uno a lo suyo por la lógica del entre todos lo haremos" (Brugué, 2005: 4).

Una herramienta importante a considerar en la participación colaborativa son las TIC, mejor conocidas como Web 2.0, las cuales demuestran que la gente está bien informada de todo y que, cuando se le da la oportunidad de reunirse en una red y en grupos, pueden ser eficaces en la solución de problemas y no sólo opinar sobre los mismos. Esta afirmación nos deja claro lo que Noveck (2010) ha planteado: la colaboración es una forma de participación democrática que es igualitaria pero diferente a la concepción tradicional del término. Y son los cambios generados por los procesos de globalización los que han conformado una sociedad en red y han dado como resultado una lógica diferente de la participación basada en la colaboración (Castells, 2008; 1999).

El hecho de que la gente esté bien informada la hace dispuesta a participar con sus habilidades, conocimientos, experiencias, información y entusiasmo para el apoyo de la gestión y políticas públicas; por lo tanto, una gestión innovadora establecerá los mecanismos e instituciones inteligentes para hacer uso de la tecnología y conectarse con los ciudadanos, haciendo de este modo de la colaboración una participación abierta, "que no es más que un tipo de participación pública a través de herramientas de comunicación interactivas como blogs, microblogs o redes sociales. Se basa principalmente en la expresión de medios sociales para conectar a la gente y ayudar a compartir sus ideas" (Cusba, 2012: 119).

Esta nueva generación tecnológica (Web 2.0), que se crea a través del uso de estos medios sociales se fundamenta en, al menos, tres pilares necesarios para entender el nuevo papel de dicha generación de tecnologías dentro de las administraciones públicas:

Por un lado, los individuos se convierten en protagonistas de la creación, organización, edición, combinación, intercambio, comentario y evaluación de contenidos web; por otro lado, en un entorno Web 2.0, la innovación en el sector público está ligada al 
público que se encuentra fuera de los límites formales de la organización; y en tercer lugar, la Web 2.0 denota una transformación en los procesos de creación de valor, ya que las personas pueden llevar a cabo muchas actividades que previamente requerían la intermediación de un profesional, un burócrata, una empresa, un partido político, etcétera (Criado, 2013: 3).

Es aquí justamente donde se requiere entender el significado del capital informacional fundamental para la articulación de una plataforma de innovación de la gestión. Entendemos a este capital como una herramienta para agilizar la innovación de la gestión pública sustentada en saberes cívicos, pues analiza datos relacionales en la gestión pública como un vínculo específico existente entre los tipos de información generados (vía presencial o virtual), y el rol de los actores que se involucran en ella. En este sentido, se caracteriza tanto por el papel jugado por los ciudadanos en el proceso de apropiación como por el funcionamiento institucional de las redes.

De lo anterior derivamos la siguiente pregunta, ¿cuáles son entonces los efectos democratizadores y cívicos del capital informacional? En primer lugar, la información se hace pública en el sentido de que se articulan datos convertidos en valor público. Se trata de esta manera de una relación transparente entre ciudadanos y gobierno donde éste orienta su trabajo hacia lo que más le importa a la gente, incorporándolo, en consecuencia, al proceso interactivo de los servicios públicos. El segundo efecto democratizador es que la información en red da cabida a que los ciudadanos y actores no gubernamentales colaboren en el proceso de hechura de la política pública y la decisión, a la vez que puedan tener voz para exigir rendición de cuentas a la autoridad (Mariñez, 2015; 2009).

Vista de esta manera, la participación colaborativa está más comprometida con lo cívico que la visión participacionista clásica que se desconecta de la hechura de las decisiones y de las políticas públicas. Así, la participación pública colaborativa es un saber cívico que sirve para alcanzar los objetivos de la innovación en la gestión pública, sirviendo como medio para la "redistribución del poder que permite a los ciudadanos, actualmente excluidos de los procesos políticos y económicos, a que se incluyan deliberadamente en el futuro" (Harrison et al., 2011: 4).

Entendido de esta forma, Vigoda (citado por Dougherty et al., 2011) sugiere:

la necesidad de un modelo mejorado de colaboración en el que los ciudadanos y los administradores públicos sean socios. Bajo el modelo ciudadano como socio, las agencias del gobierno son responsables de definir los objetivos estratégicos y el empoderamiento 
de los ciudadanos a través de la formación y la coordinación de las actividades voluntarias ciudadanas (Dougherty et al., 2011: 522).

\section{Procedimiento de la participación colaborativa para la activación de los saberes cívicos}

La nueva lógica de la participación colaborativa requiere entonces de la conexión, el diálogo, la asociación y el intercambio para poder construir decisiones y políticas públicas legítimas. Se trata de una participación en el marco de una nueva forma de relación entre gobierno y ciudadanos que necesita de un procedimiento distinto, implicando para ello la solidez de instituciones para una gestión pública con saberes cívicos (véase Cuadro 2).

Pero para el logro de esta solidez de las instituciones son necesarios desafíos como la construcción de los procesos técnico, institucional y político, donde las amenazas emergentes y las oportunidades juegan un factor relevante. Según Bardach (2008), el desafío técnico cuenta con la adquisición de recursos, el diseño y la ejecución de un sistema operativo (es decir, un sistema para realizar tareas a nivel de la base); la creación y el mantenimiento de una red de comunicaciones, y la disposición de un ambiente de (justificada) confianza; el empoderamiento y la legitimación de un proceso de dirección (o gobernanza); y un conjunto de individuos para ejecutarlo. Respecta a los desafíos institucional y político, es importante un diseño institucional que favorezca la cooperación entre las agencias (departamentos, direcciones) y entre éstas con los ciudadanos.

Con estos elementos institucionales mencionados, la participación colaborativa podría expresarse a través de una combinación de actividades incluyentes y articuladas que implica un cambio total en la cultura organizativa y administrativa de las agencias de gobiernos tanto centralizadas como descentralizadas. Una cultura de gestión innovadora en una organización pública implica coinnovación, colaboración, cocreación, transparencia, redes integradas, horizontalidad y una nueva visión institucional en las estructuras de implementación de la gestión misma y de las decisiones públicas, basada en la argumentación, los modelos de comunicación y la gobernanza (Peters, 2014).

Apoyándonos en Lukensmeyer et al. (2010), identificamos tres categorías participativas donde están incluidas las diferentes actividades de participación colaborativas:

1. Participación en línea. Ésta se caracteriza porque el compromiso de los ciudadanos en los planes del gobierno se involucra más y más con el uso 
de herramientas basadas en TIC, a través del crowdsourcing o ideas, los concursos ciudadanos, los Wikis, las Salas abiertas online para los chats entre ciudadanos y funcionarios, y los medios de comunicación.

2. Participación cara a cara. Una participación con visión colaborativa debe incluir actividades donde a través del método cara a cara se puedan construir consensos entre los ciudadanos, stakeholders y funcionarios públicos. En este sentido, los tipos de participación más utilizados son las sesiones de audiencias públicas: los foros dirigidos a los stakeholders y los foros ciudadanos.

3. Participación formal. Las reglas y regulaciones gubernamentales prevén varios mecanismos formales mediante los cuales las oficinas gubernamentales deben consultar al público para solicitar insumos y participación. Una oficina pública con una estrategia inclusiva se refiere a menudo a este tipo de participación con información sobre los programas en curso donde han participado ciudadanos o grupos de interés en un año determinado, utilizando canales tradicionales para la consulta del público.

En este sentido, dos formas de participación son los más comunes: por un lado, los consejos consultivos ciudadanos, y por el otro, los esquemas de producción de reglas.

Es importante destacar que estas formas de participación colaborativa están profundamente vinculadas tanto con el compromiso cívico como con las habilidades de los funcionarios públicos y la inteligencia cívica de los ciudadanos. En cuanto a los funcionarios públicos, "lo importante es que quien representa una organización, agencia o jurisdicción en la administración pública deba tener las habilidades necesarias para ser un colaborador eficaz" (O’Leary y Vij, 2012: 515). Y al referirnos a la inteligencia cívica damos cuenta de los diversos tipos de proyectos ciudadanos y comunitarios para motivar y coordinar múltiples esfuerzos de innovación pública y social.

En este sentido, para una innovación, Eggers (2009) propone un conjunto de competencias y habilidades con una visión de colaboración para los funcionarios públicos: habilidad de asociarse, influencia y negociación, habilidades interpersonales, creatividad e innovación, conciencia en el contexto externo, emprendedor, solucionador de conflictos y manejo de conflictos. De esta manera, los funcionarios, sobre todo de carrera, tendrían la capacidad para el diseño de estructuras, procesos, prácticas, normas de comunicación, patrones y demás elementos de la gestión de la innovación en el gobierno, por lo que hoy no serían obstáculos para un gobierno inclusivo y en red, sino que serían actores clave en este proceso (Fountain, 2005). 


\section{A manera de conclusión}

El artículo abordó, desde el inicio, la importancia de los saberes cívicos en la innovación de la gestión pública. Dejamos claro que el centro de análisis de la innovación en la gestión pública debe ir de la mano tanto del fortalecimiento democrático como de los saberes cívicos y societales de los ciudadanos en relación con el gobierno. Así, estos saberes se convierten en los aspectos sustantivos de la innovación gubernamental, porque tanto el compromiso cívico como la inteligencia cívica, el control social y la participación colaborativa logran fortalecer el emprendimiento que implica la necesidad de habilidades y competencias generadoras de capacidades para resolver problemas públicos, aportando un valor añadido a los arreglos del sector público en el que una gran cantidad de talentos y esfuerzos que trabajan en y cerca de la administración pública están disponibles para tratar de cumplir con estos desafíos.

Sustentado en los objetivos del artículo explicitados en la introducción y haciendo una revisión teórica para ver cómo se ha discutido el compromiso cívico, la inteligencia cívica, el control social y, sobre todo, la participación colaborativa, logramos construir un marco de interacciones de estos conceptos, así como la manera en que se relacionan con la innovación en la gestión pública.

En este sentido, hemos concebido a la innovación en la gestión pública en el marco de dos direcciones: una, a los arreglos de los ciudadanos, actores gubernamentales y no gubernamentales que permiten identificar problemáticas públicas, así como herramientas y dispositivos novedosos para el logro de respuestas y soluciones nuevas en el espacio de lo público. Dos: como consecuencia de la anterior, a las modalidades democráticas de articulación de estos actores en la organización pública, produciendo una nueva configuración entre el Estado y la sociedad, que asegura el aprendizaje colectivo y los saberes cívicos necesarios para una gestión de calidad e inclusiva.

Así, los componentes de los saberes cívicos son las piezas democráticas fundamentales para el logro de una innovación en la gestión pública participativa. En cuanto al compromiso cívico, hemos partido de que una innovación participativa de la gestión pública requiere de diversas formas de diálogo y deliberación ciudadano que son altamente inclusivas para la hechura de las políticas y decisiones públicas. En este sentido, la inclusión es un aspecto importante cuando hablamos del compromiso cívico, pues tiene como objetivo proporcionar a las personas un sentido de dirección para que las actividades asociativas y comunitarias tengan voz en su relación con el gobierno y así ayudarle a mejorar sus decisiones. Se trata de un punto esencial de la parti- 
cipación ciudadana que asegura que todos los ciudadanos hagan uso de sus derechos cívicos, políticos y sociales.

Para que el compromiso cívico se realice es importante que los ciudadanos activen la inteligencia cívica, otro componente de los saberes cívicos, ya que pone la capacidad de los ciudadanos y grupos de la sociedad civil para concebir e implementar estrategias eficaces, equitativas y sostenibles para compartir problemas y soluciones públicos. En este sentido, los ciudadanos aprovechan las herramientas Web 2.0 para adquirir recursos, conocimientos, experiencias o tiempo (colaboración abierta) para la cocreación de decisiones públicas. De allí lo cívico de la inteligencia cívica para la innovación en la gestión pública.

Partimos de que las innovaciones en la organización, en los procesos y en el aprovisionamiento de los servicios públicos deben ser monitoreadas, vigiladas y evaluadas por los ciudadanos y sus organizaciones. Hemos insistido que a través del software social se logra una serie de aplicaciones que pretenden ampliar las posibilidades de comunicación y conexión entre personas que ya permiten el e-mail o los sistemas de mensajería instantánea. Son herramientas colaborativas que unen a una serie de personas con el fin de facilitar el flujo de información (agendas, mensajes, denuncias) y la realización de trabajos conjuntos entre ellas (colaboración en el monitoreo o vigilancia de las políticas públicas).

En este sentido propusimos cuatro ejes que hacen al control social más efectivo: la transparencia colaborativa, la organización y las capacidades de los ciudadanos, el acceso a la información y el eje relacionado con lo público que implica que todos los actores que intervienen en la producción del valor público son objetos de ser controlados por sus acciones.

Lo planteado nos lleva a ubicar la participación en la lógica de la colaboración, ya que haciendo uso de la Web 2.0, los ciudadanos se convierten en protagonistas de la creación, organización, edición, combinación, intercambio, comentario y evaluación de contenidos web, así como en la formación de redes sociales donde interactúan y se enlazan entre ellos. También en el entorno de Web 2.0, la innovación en la gestión pública está ligada al público que se encuentra fuera de los límites formales de la organización, que tiene la capacidad no sólo para generar información y contenidos, sino también para evaluarlos de manera continua. Y otro elemento aprovechado por la participación colaborativa es que a través de la Web 2.0 se denota una transformación en los procesos de creación de valor, ya que las personas pueden llevar a cabo muchas actividades que previamente requerían la intermediación de un profesional, un burócrata, una empresa, un partido político, etcétera. 
Con una gestión innovadora en esta dirección, se activarán otros ejes democratizadores tales como la transparencia, la rendición de cuentas y el acceso a la información. Pero como se precisó en el desarrollo del trabajo, se requieren desafíos como la construcción del proceso técnico, institucional y político para darle respuesta a las amenazas y oportunidades emergentes que sirvan de elementos articuladores activos entre los ciudadanos, actores gubernamentales y no gubernamentales. Son estos elementos los que le imprimen un diferencial político sustantivo a la forma de gobernar a través de la innovación en la gestión participativa.

Observamos muchos desafíos teórico-prácticos en la innovación en la gestión pública basada en los saberes cívicos. El éxito de este tipo de innovación está relacionado con el grado en la que toda la organización está dirigida a ella, y no sólo a una parte. Esto lo lleva a plantearnos las siguientes interrogantes: ¿qué tipo de organizaciones públicas son innovadoras y quiénes son los conductores de las innovaciones en las organizaciones públicas?, ¿cómo el sector público innova las organizaciones y cómo acceden y usan el nuevo conocimiento generado en la coproducción y la cocreación? ¿Qué funcionarios públicos requiere la administración pública para estructurar, organizar y promover la innovación en la gestión pública? En las administraciones públicas y gobiernos que portan aún rasgos de patrimonialismo, falta de profesionalización y opacidad, estas tendencias innovadoras son empujadas por los saberes cívicos como prácticas sociopolíticas-administrativas.

\section{Bibliografía}

Agranoff, R. y M. McGuire (2003), Collaborative public management: New strategies for local governments, Washington, DC: Georgetown University Press.

Aguilar Villanueva, Luis (2006), Gobernanza y gestión pública, México: Fondo de Cultura Económica.

Bailur, Savita y Bjorn-Soren Gigler [eds.] (2014), Closing the Feedback Loop. Can Technology Bridge the Accountability Gap?, Washington, DC: The World Bank.

Barber, Benjamin (2004), Democracia fuerte. Politica participativa para una nueva época, España: Almuzara.

Bardach, Eugene (2008), “Developmental Processes: A Conceptual Exploration”, en Innovations in government. Research, Recogniyion and Replication, Washington DC: Harvard University, Kennedy School of Government, Brookings Institution Press.

Bott, Maja et al. (2014), "The rol of Crowsourcing for better Governance in Fragile State Contexts", en Savita Bailur y Bjorn-Soren Gigler [eds.], Closing the Feedback Loop. Can Technology Bridge the Accountability Gap?, Washington, DC: The World Bank. 
Brainard, Lori A. y John G. McNutt (2010), "Virtual Government- Citizen Relations: Informational, Transactional, or Collaborative?", en Administration \& Society, Virginia Tech, USA: Sage Publications.

Brugué, Quim (2005), Modernizar la administración. Burocracia, Nueva gestión pública y administración deliberativa, Barcelona: IGO-UAB (mimeo).

Castell, Manuel (2008), "The New Public Sphere: Global Civil Society, Communication Networks and Global Governance", en The Annual of The America Academy of Political and Social Science, núm. 616, marzo, Philadelphia, USA: Sage Publications, University of Pennsylvania.

Castell, Manuel (1999), La era de la información. La sociedad red, vol. 1, España: Siglo XXI. Carta Iberoamericana de Participación Ciudadana en la Gestión Pública (2009), Revista del CLAD Reforma y Democracia, núm. 45, México.

Criado Grande, J. Ignacio (2013), "Redes sociales para unas administraciones y gobiernos abiertos: desafíos para la innovación y la creación de valor público", XVIII Congreso Internacional del CLAD sobre la Reforma del Estado y de la Administración Pública, 29 de octubre- 1 de noviembre, Montevideo, Uruguay.

Cusba, Enrique (2012), "Colaboración: El gobierno en doble vía con las personas", en Gastón Concha y Alejandra Nasser [coords.], El desafio en el gobierno abierto en la hora de la igualdad, Documento de proyecto, Chile: CEPAL.

Dougherty, Jr., George W. y Jennifer Easton (2011), "Appointed Public Volunteer Boards: Exploring the Basics of Citizen Participation Through Boards and Commissions", en The American Review of Public Administration, vol. 41, núm. 5, Columbia, USA.

Dryzek, John S. (2009), "Democratization as Deliberative Capacity Building", en Comparative Political Studies. Sage Journals, vol. 42, núm. 11, Minneapolis, USA: University of Minnesota.

Edelmann, Noella et al. (2012), "Collaboration for Open Innovation. Process in Public Administration", en Y. Charalabidis y S. Koussouris [eds.], Empowering Open and Collaboration Governance, Berlín Heidelberg: Springer-Verlag.

Eggers, William (2009), "From conflict to Collaboration: Lessons in Networked Governance from the Federal Cooperative Conservation Initiative", en Stephen Goldsmith y Donald F. Kettl [eds.], Unlocking the Power of Networks. Keys to High-Performance Government, Washington DC: ASH Institutte for Democratic Governnance and Innovation/JFK School of Government, Harvard University.

Fountain, Jane (2005), "Central Issues in the Political Development of the Virtual State", en The Network Society and the Knowledge Economy: Portugal in the Global Context, 4-5 de marzo, Lisboa.

Fung, Archon (2008), "Citizen Participation in Government Innovations", en Sandford Borins [ed.], Innovations in Government. Research, Reconition and Replication, USA: Institute for Democratic Governance and Innovations y Harvard University.

Garcés Lloreda, María Teresa (2006), "La Veeduría Distrital y la Política Bogotá Transparente: un modelo de control preventivo y de liderazgo ético para una gestión de integridad en el Distrito Capital", en Ernesto Isunza Vera y Alberto J. Olvera, Democratización, 
rendición de cuentas y sociedad civil: participación ciudadana y control social, México: CIESAS, Universidad Veracruzana y Miguel Ángel Porrúa.

Goldsmith, Stephen et al. (2010), The Power of Social Innovations. How civic entrepreneurs ignite community Networks for Good, USA: Jossey-Bass.

Harrison, Teresa M et al. (2011), "Open Government and E-Government: Democratic Challenges from a Public Value Perspective", en The Proceeding of the 12th Annual International Digital Government Research Conference.

Harrison, Denis y Juan-Luis Klein (2007), "Introduction”, en Denis Harrison y Juan-Luis Klein [coords.], L'Innovation sociale. Émergence et effets sur la transformation des societies, Canadá: Presses de l'Université du Québec.

Hevia de la Jara, Felipe (2006), La Contraloría Social mexicana: Participación ciudadana para la rendición de cuentas. Diagnóstico actualizado a 2004, México: Centro de Investigaciones y Estudios Superiores en Antropología Social (CIESAS), Instituto de Investigaciones Histórico Sociales, Universidad Veracruzana.

Im, Tobin y Seung Jong Lee (2011), "Does Management Performance Impact Citizen

Satisfaction?”, en The American Review of Public Administration, vol. 42, núm. 4, Columbia, USA.

LaDuke, Bruce (2008), "Knowledge creation in collective intelligence", en Mark Tovey [ed.], Collective Intelligence: Creating a prosperous World at Peace, USA: Earth Intelligence Network (EIN).

Lukensmeyer, Carolyn et al. (2010), Assessing Public Participation in an Open Government Era. A Review of Federal Agency Plans, USA: IBM Center for The Business of Government, Fostering Transparency and Democracy Series.

Mariñez Navarro, Freddy (2015), Innovación pública. Para que funcionarios y ciudadanos actúen con saberes civicos, México: Fontamara.

Mariñez Navarro, Freddy (2009), "El Capital Informacional en la Gestión Pública. Una aproximación al análisis de redes", en Revista Enfoques. Ciencia Politica y Administración Pública, vol. VII, núm. 11, Santiago de Chile.

Meijer, Albert et al. (2012) “Open government: connecting vision and voice”, en International Review of Administrative Sciences, marzo, vol. 78, núm. 1, Bruselas, Bélgica.

Michels, Ank (2011), "Innovations in democratic governance: how does citizen participation contribute to a better democracy?", en International Review of Administrative Sciences, Sage Journals, junio, vol. 77, núm. 2, Bruselas, Bélgica.

Mothae, Lebohang y Moses Sindane (2013), "Leadership, Governance and Public Policy Competencies in the broader public sector", en Michiel S. de Vries y Geert Bouckaert [eds.], Training for Leadership, Bruylant-IASIA-IIAS.

Mulgan, Geoff y David Albury (2003), Innovation in the public sector, London: Cabinet Office.

Noveck, Beth Simone (2010), "The Single Point of Failure", en Daniel Lathrop y Laurel Ruma [eds.], Open Government. Collaboration, Transparency and Participation in Practice, USA: O'Reilly. 
Saxton, Gregory D. y Chao Guo (2011), "Accountability Online: Understanding The Web-Based Accountability Practices of Nonprofit Organizations”, en Nonprofit and Voluntary Sector Quartely, vol. 40, núm. 2, Philadelphia, USA: University of Pennsylvania.

Seltzer Ethan y Dillon Mahmoudi (2012), “Citizen Participation, Open Innovation, and Crowdsourcing: Challeges and Opportunities for Planning”, en Journal of Planning Literature, vol. 28, núm. 1, USA: The Ohio State University.

Shuler, Douglas (2010), "Online Deliberation and Civic Intelligence", en Daniel Lathrop y Laurel Ruma [eds.], Open Government, Collaboration, Transparency and Participation in Practice, USA: O'Reilly.

Shuler, Douglas (2008), "Civic intelligence and the public sphere”, en Mark Tovey, Collective Intelligence: Creating a Prosperous World at Peace. The Wealth of Networks: Remixed Highlights, USA: Earth Intelligence Network.

Simrell King, Sheryl y Alicia Seegers Martinelli (2005), "Innovations in Citizen Engagement and Empoerment: Beyond Boudaries", en The innovation Journal: The Public Sector Innovation Journal, vol. 10, núm. 1, Canada: Simon Fraser University.

OCDE (2012), La Estrategia de Innovación de la OCDE. Empezar hoy el mañana. OCDE, Foro Consultivo Científico y Tecnológico, México.

O’Leary, Rosemary y Nidhi Vij (2012) “Collaborative Public Management: Where Have We Been and Where Are We Going?", en The American Review of Public Administration, vol. 42, núm. 5, Columbia, USA.

Peters, B. Guy (2014), "Implementation structures as institutions", en Public Policy and Administration, Sage Journals, USA: Oregon State University.

Rizvi, Gowher (2008), "Innovations in Government: Serving Citizens and Strenhening Democracy”, en Sandford Borins, Innovations in government. Research, Recognition and Replication, USA: Ash Institute for Democratic Governance and Innovation, Harvard University.

Wildrum, P. (2008), "Innovation and Entrepreneuriship in Public Services", en Wildrum, P. y P. Koch [eds.], Innovation in Public Sector Services, Cheltenham: Edward Elgor.

Zurbriggen, Cristina y Mariana González Lago (2014), "Innovación y co-creación. Nuevos desafíos para las políticas públicas”, en Revista de Gestión Pública, vol. III, núm. 2, Santiago, Chile.

\section{Recurso electrónico}

Subirats, Joan (2015), “Modernidad y protagonismo ciudadano", en El País, 5 de abril. Disponible en: www.elpais.com [5 de abril de 2015]. 


\section{Anexo}

\section{Cuadro 1}

\section{Tipos de innovación en la gestión pública}

\begin{tabular}{|c|c|c|}
\hline Características & Innovación con saberes cívicos & Innovación gerencial \\
\hline $\begin{array}{l}\text { Tipos de } \\
\text { satisfacciones }\end{array}$ & $\begin{array}{l}\text { Satisfacción de los ciudadanos. } \\
\text { Comunicación entre funcionarios } \\
\text { y ciudadanos para la innovación. } \\
\text { El proceso es tan importante } \\
\text { como los resultados, y la confianza } \\
\text { pública se basa en la democracia. }\end{array}$ & $\begin{array}{l}\text { Satisfacción del cliente. } \\
\text { Mejora continua de los } \\
\text { procesos, productos y } \\
\text { servicios. }\end{array}$ \\
\hline Aprendizajes & $\begin{array}{l}\text { Aprendizaje colectivo } \\
\text { (funcionarios y ciudadanos). } \\
\text { Relación entre las condiciones } \\
\text { internas y externas de la } \\
\text { organización. }\end{array}$ & $\begin{array}{l}\text { Aprendizaje colectivo. } \\
\text { Sólo entre funcionarios. }\end{array}$ \\
\hline Tipo de organización & Inclusiva, flexible, relacional & $\begin{array}{l}\text { Organización relacional } \\
\text { no inclusiva }\end{array}$ \\
\hline $\begin{array}{l}\text { Participación en la } \\
\text { gestión }\end{array}$ & $\begin{array}{l}\text { Gestión pública participativa. } \\
\text { Los funcionarios públicos crean } \\
\text { oportunidades para comprometer } \\
\text { a los ciudadanos, usando estas } \\
\text { oportunidades para educar, } \\
\text { organizar y activar la defensa de la } \\
\text { participación ciudadana. }\end{array}$ & $\begin{array}{l}\text { Gestión pública no } \\
\text { participativa. } \\
\text { Gerencialismo y un marco } \\
\text { orientado al mercado en } \\
\text { la entrega del servicio } \\
\text { público o producto. }\end{array}$ \\
\hline $\begin{array}{l}\text { Criterios de } \\
\text { legitimidad }\end{array}$ & $\begin{array}{l}\text { Motivaciones democráticas para } \\
\text { participar en la cocreación de la } \\
\text { innovación del servicio público. }\end{array}$ & $\begin{array}{l}\text { Eficacia y eficiencia en los } \\
\text { procesos. }\end{array}$ \\
\hline
\end{tabular}

Fuente: Elaboración propia. 


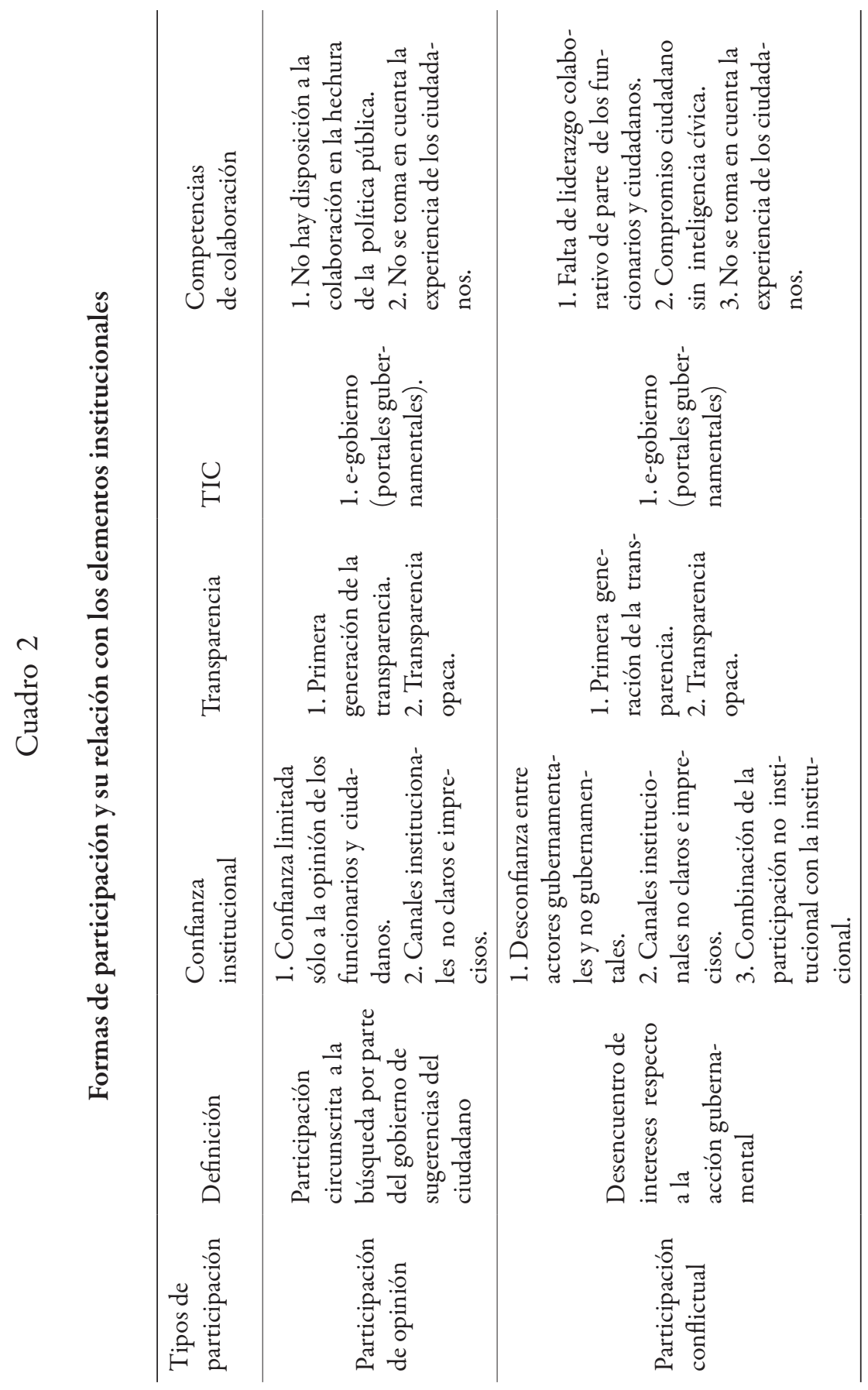




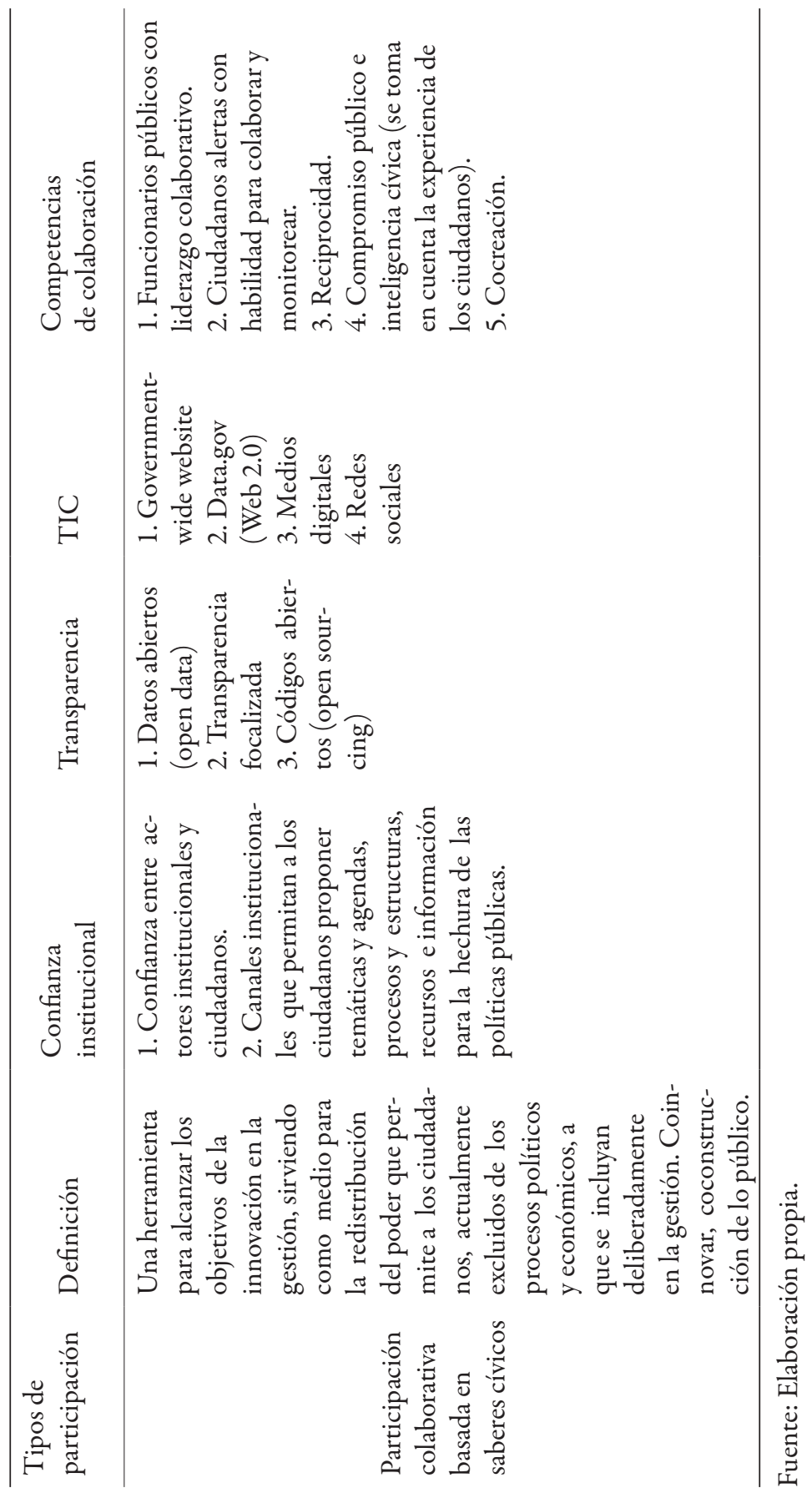


Freddy Mariñez Navarro. Ph. D en Sociología Política por la Université Laval, Canadá. Especialista en política pública, gestión pública, gobernanza e innovación pública. Es profesor investigador del Doctorado en Estudios Humanísticos del Tecnológico de Monterrey (Campus Monterrey), México. Miembro del Sistema Nacional de Investigadores del Conacyt, México, Nivel II. Líneas de investigación: gestión pública, políticas públicas, gobernanza y participación de la sociedad civil. Publicaciones recientes: Innovación Pública. Para que funcionarios y ciudadanos actúen con saberes cívicos, México: Fontamara (2015); en coautoría con José Fernández Santillán, La perspectiva internacional de los partidos politicos en México, México: Fontamara (2014); en coautoría con Rafael Valenzuela Mendoza, Gobierno Abierto: ¿más innovación?, ¿más gobierno?, ¿más sociedad?, ¿en qué consiste? Propuestas para los gobiernos locales, México: Miguel Ángel Porrúa (2013); Hagamos entre todos la política pública. Una reflexión sobre la visión relacional de la politica pública, México: Miguel Ángel Porrúa (2011).

Recepción: 23 de octubre de 2014.

Aprobación: 14 de septiembre de 2015. 